\title{
Diagnostic Hysterolaparoscopy in the Evaluation of Female Factor Infertility: A Cross-sectional Study at a Tertiary Care Hospital in Central India
}

\author{
Prashanthi Selvaraj $^{1}$, Moushmi Parpillewar ${ }^{2}$, Juzar Fidvi ${ }^{3}$
}

\begin{abstract}
Background: Infertility is a worldwide problem affecting people of all geographical location and socioeconomic status. It is estimated that globally 60-80 million couples suffer from infertility every year. The main objective of the study is to detect the diagnostic efficacy of hysterolaparoscopy in evaluating the tubal, uterine, ovarian, and pelvic pathologies causing infertility and also compare the findings of a hysterosalpingogram with diagnostic laparoscopy in evaluating the tubal factor infertility.

Materials and methods: This hospital-based cross-sectional study included an evaluation of 1,456 couples presenting with complaints of infertility at the Outpatient Department of Government Medical College, Nagpur during the period of February 2016 to September 2018. After thorough gynecological examination and investigations, 130 infertile women were posted for diagnostic hysterolaparoscopy. All the data were collected on predesigned proforma and the results were tabulated and raw percentages calculated to describe the results.

Results: Among the 130 infertile women, 102 women (78.46\%) had primary and 28 (21.53\%) had secondary infertility. The maximum number of patients belonged to the age group of $26-30$ years (39.23\%). About $56 \%$ of patients were in the active marriage life of 3-5 years duration. Most of them had multiple factors affecting their fertility. Out of the 130 infertile women, 32 patients (24.62\%) had uterine factors, 44 patients (33.84\%) had peritoneal factors, 67 patients (51.5\%) had ovarian factors, and 89 patients $(68.46 \%)$ had tubal factors. Only eight patients (6.15\%) had normal findings in laparoscopy. Out of the 130 patients, 122 (93.84\%) had more than one factor causing infertility. About $26.15 \%$ of patients had positive findings in diagnostic hysteroscopy.

Conclusion: Hysterolaparoscopy provides both a panoramic view of the pelvic reproductive anatomy and also a magnified view of the pelvic organs and peritoneal surfaces. It is generally regarded as the most reliable tool in the diagnosis of tubal pathology and other intra-abdominal causes of infertility because of the correctable abnormalities like endometriosis, adnexal adhesions, and septate uterus which are usually missed, are usually identified that helps in formulating a specific plan of management.
\end{abstract}

Keywords: Diagnostic laproscopy, Hysteroscopy, Infertilty.

International Journal of Infertility and Fetal Medicine (2020): 10.5005/jp-journals-10016-1203

\section{INTRODUCTION}

The World Health Organization defines health as a state of complete physical, mental, and social well-being and not merely the absence of disease. By this definition, infertility is a major cause of diminished health in both developing and developed countries. ${ }^{1}$ The prevalence of infertility ranged from 3.5 to $16.7 \%$ in developed and 6.9 to $9.3 \%$ in developing nations. ${ }^{2}$ Before the advent of hysterolaparoscopy, infertile females were evaluated for tubal factor by hysterosalpingography (HSG), ovarian factor by hormone profile or folliculometry, and uterine factor by transvaginal ultrasound. But now, the advanced endoscopic instruments demonstrate the superiority of direct visualization over the radiographic examination of various body cavities. The ability to observe and treat during laparoscopy has made this procedure a gold standard technique to evaluate pelvic pathology. Similarly, visualizing the uterine cavity and identifying the possible pathology and treating at the same time has made hysteroscopy an essential part of an infertility evaluation. Thus, it becomes a diagnostic as well as a therapeutic procedure. The focus of infertility treatment has shifted from the systematic correction of each identified factor in applying the most efficient therapy. The investigation of infertile couples should be rapid and inexpensive, using a minimally invasive test. However, because of the cost and slightly invasive nature of laparoscopy, it should not be the initial test in the couple's diagnostic evaluation. The routine use
${ }^{1-3}$ Department of Obstetrics and Gynaecology, Government Medical College, Nagpur, Maharashtra, India

Corresponding Author: Prashanthi Selvaraj, Department of Obstetrics and Gynaecology, Government Medical College, Nagpur, Maharashtra, India, Phone: +919403397882, e-mail: swaha_dundoo@yahoo.com

How to cite this article: Selvaraj P, Parpillewar M, Fidvi J. Diagnostic Hysterolaparoscopy in the Evaluation of Female Factor Infertility: A Cross-sectional Study at a Tertiary Care Hospital in Central India. Int J Infertil Fetal Med 2020;11(2):37-41.

Source of support: Nil

Conflict of interest: None

of diagnostic laparoscopy for the evaluation of all cases of female infertility is currently under debate. According to data published in retrospective non-controlled studies, diagnostic laparoscopy after several failed cycles of ovulation induction enables the detection of a significant proportion of pelvic pathology amenable to treatment. It has been estimated that using laparoscopy as a standard test, will reduce the apparent incidence of unexplained infertility from 10 to $3.5 \%{ }^{3}$ This study was undertaken to evaluate the role of diagnostic hysterolaparoscopy in the comprehensive workup of infertility, which would help in planning appropriate management and also compare the findings of HSG and diagnostic laparoscopy in evaluating the female factors infertility. 


\section{Materials and Methods}

This study is a hospital-based cross-sectional study done at a tertiary care center in Central India, done from February 2017 to September 2019 for a period of 18 months. A total of 1,456 infertile couples attending the infertility clinic at Government Medical College, Nagpur were recruited in the study. The screening tests included ultrasonography (USG) of the pelvis, semen analysis, HSG, Chlamydia antibody testing, thyroid function test, and hormonal testing, in selected patients. Out of them, 130 infertile women were recommended diagnostic hysterolaparoscopy as the next line of investigation in their infertility workup, and findings were recorded in a structured proforma.

\section{Primary Objective}

To find out the role of diagnostic hysterolaparoscopy in the evaluation of different factors causing female infertility and the feasibility of providing therapeutic management concurrently.

\section{Secondary Objective}

To compare the findings of HSG with findings on diagnostic laparoscopy with chromopertubation in the evaluation of tubal factor infertility.

\section{Inclusion Criteria}

- Women with primary and secondary infertility.

- Women with failed ovulation induction in three cycles.

- Abnormal HSG findings.

\section{Exclusion Criteria}

- Medical disorders are a contraindication for anesthesia.

- Active pelvic infection.

- Couple with any psychosexual dysfunction.

- Couple under any kind of substance abuse.

Detailed history, physical examination, basic endocrinological investigations, USG pelvis (preferably transvaginal), HSG, and semen analysis were done before the procedure. Hysterolaparoscopy was done under appropriate anesthesia between days 9 and 12 of the menstrual cycle. Chromopertubation was carried out in all cases to test the patency of the tube under laparoscopic vision by using 15 $\mathrm{mL}$ of $0.5 \%$ methylene blue dye.

\section{Statistical Analysis}

Data were coded and analyzed in statistical software, STATA version 10.1, 2011. Descriptive statistics included summary measures like mean and standard deviation for quantitative variables and frequency, percentages for categorical variables.

\section{Results}

The demographic parameters are given in Table 1. Out of the 130 women, 102 (78.4\%) had primary infertility and 28 (21.53\%) had secondary infertility. Female factor infertility accounted for 91 cases $(70 \%)$, male factor in 13 cases (10\%), both in 19 cases $(14.6 \%)$, and unexplained infertility in 7 cases (5.38\%) (Fig. 1). Nearly $56 \%$ reported at our infertility clinic was in the active marriage life of 3-5 years, while only about $3 \%$ of couples had infertility of $>12$ years (Fig. 2). Out of the 130 patients, 67 patients (51.5\%) had ovarian findings on laparoscopy, tubal factors were seen in 89 patients $(68.46 \%), 55$ patients $(42.30 \%)$ had peritoneal factors, and
Table 1: Demographic profile of patients

\begin{tabular}{lll}
\hline Parameter & Number of cases $(n)$ & Percentage \\
\hline Total no. of cases & 130 & \\
Primary infertility & 102 & 78.6 \\
Secondary infertility & 28 & 21.5 \\
Age group-majority & $26-30$ years & 51.5 \\
Mean age & 27.93 years & \\
Marital life-majority & $3-5$ years & 56 \\
BMI-majority & $81(18.5-24.9)$ & 62.3 \\
\hline
\end{tabular}

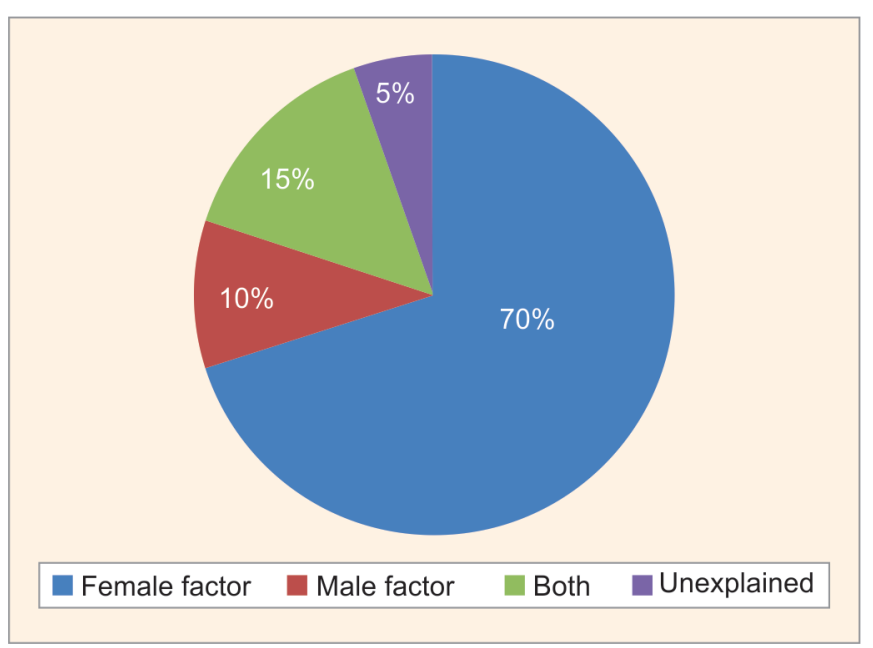

Fig. 1: Distribution of subjects according to different factors affecting the fertility of a couple

21 patients (16.15\%) had uterine factors (Fig. 3). Many patients had more than one factor responsible for their infertility which was diagnosed by laparoscopy. Out of 130 patients, no abnormality was found in 7 patients (5.38\%) and all four factors were found in 1 patient $(0.77 \%)$.

A tubal factor was the most commonly encountered abnormality in laparoscopy. Diagnostic hysteroscopy was done in all cases along with diagnostic laparoscopy. Out of the 130 patients, 96 cases $(78.85 \%)$ had no abnormality in hysteroscopy. Septate uterus (13 cases, $10 \%$ ) was the most common abnormality. Various therapeutic interventions were done in selected patients during diagnostic laparoscopy (Fig. 4) and hysteroscopy (Fig. 5). Polycystic ovarian drilling was the most common therapeutic procedure attempted through laparoscopy and septum resection through hysteroscopy. Out of the 130 cases subjected to both HSG and laparoscopy, there was a difference in findings between HSG and chromopertubation in 40 cases (30.77\%). Only in about 90 cases $(69.23 \%)$, the findings of HSG correlated with laparoscopy. Hence, tubal factor infertility cannot be confirmed with HSG alone. Diagnostic laparoscopy with chromopertubation can be done in cases where tubal factor has been ruled out with HSG but still conception has not occurred for more than three cycles. A more interesting observation was that when these two modalities were combined then the diagnostic accuracy reached $100 \%$. This reinstated the age-old theory that HSG and laparoscopy are complementary and not supplementary in diagnosing tubal factor of infertility. Calculating the sensitivity, specificity, and validity of HSG is well beyond the scope of this study. 


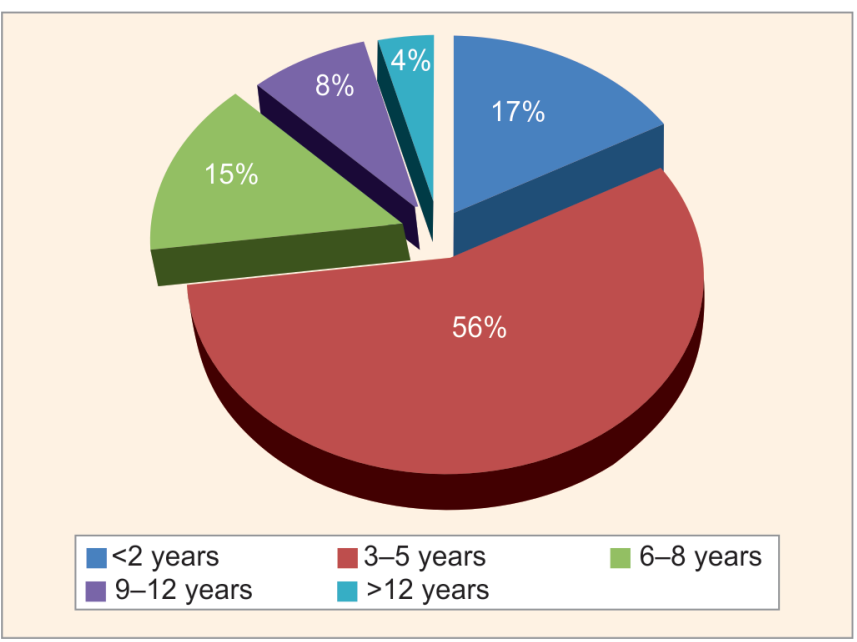

Fig. 2: Distribution of subjects according to the duration of infertility

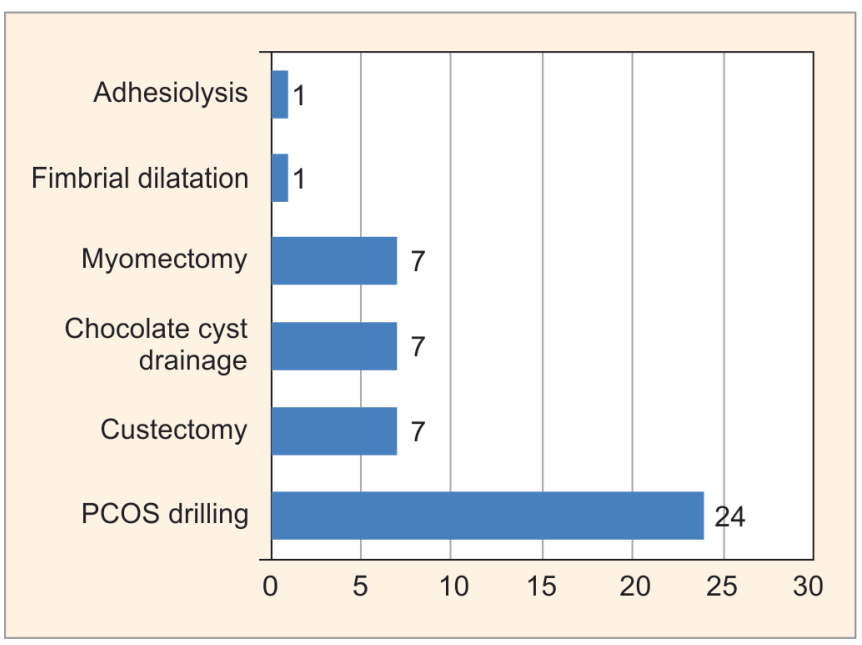

Fig. 4: Distribution of subjects according to therapeutic intervention during laparoscopy

\section{Discussion}

Exploration of the female genital tract is one of the essential elements of infertility assessment. Laparoscopy provides both a panoramic view of the pelvic reproductive anatomy and a magnified view of the peritoneal surfaces. It is generally regarded as the most reliable tool in the diagnosis of tubal pathology and other intra-abdominal causes of infertility. ${ }^{4,5}$ About $96 \%$ of women in our study were aged 20-35 years, thus supporting the fact of early marriage and childbearing in developing countries. This is in contrast to the current trend in developed as well in developing countries to some extent to defer childbearing until the mid30 s, which results in a diminished time frame available both for conception and for infertility evaluation. The predominance of a female factor was seen in both primary and secondary infertility, but the female factor was significantly higher in secondary infertility than in primary infertility. This was explained by Ngowa et al., ${ }^{6}$ where they suggested that pelvic infection resulting from ascending infection during childbirth can lead to tubal damage and thus higher contribution to the incidence in the female factors in secondary infertility.

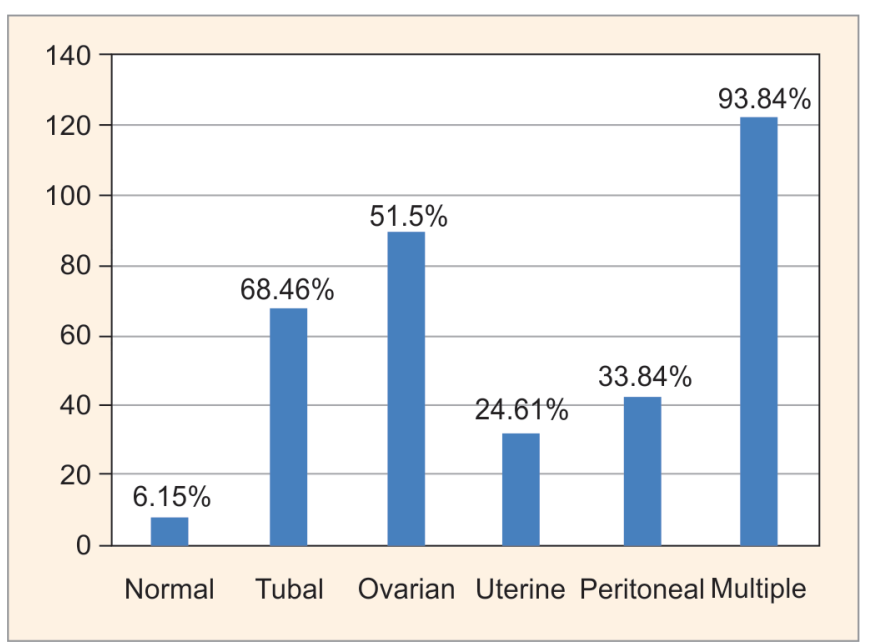

Fig. 3: Distribution of subjects according to findings at diagnostic laparoscopy

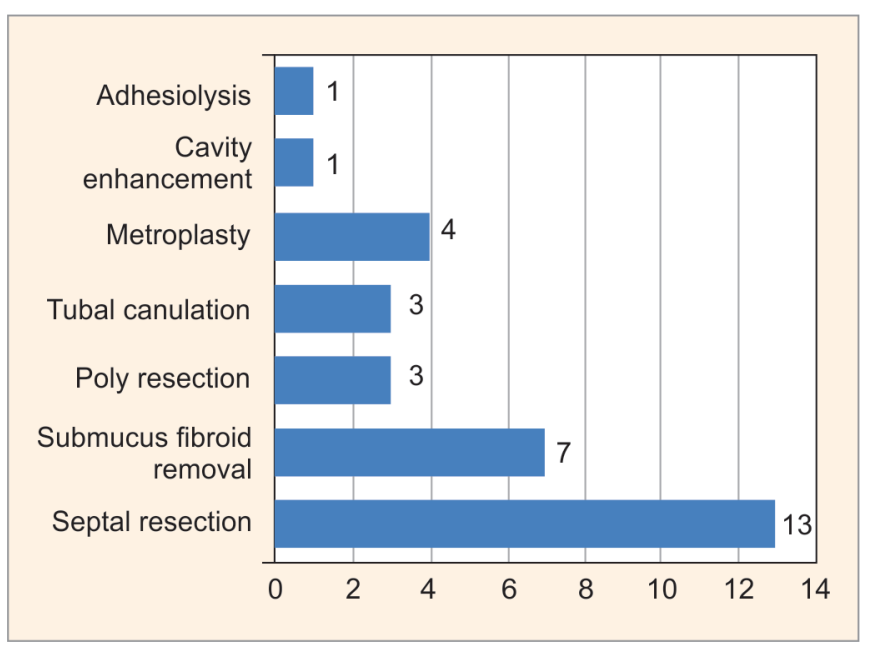

Fig. 5: Distribution of subjects according to intervention during hysteroscopy

Infertile women with normal ovulation, pelvic ultrasound findings, hormonal profile, and husband semen analysis have a higher possibility of tubo-peritoneal pathologies like endometriosis (Fig. 6). Performing hysterolaparoscopy as a 'one-step procedure' in these women will be more beneficial. There could be more than one cause of infertility in a patient and it becomes equally important to diagnose each cause as each individual cause need specific and complete treatment. In our study, an effort was made to find out the role of diagnostic hysterolaparoscopy in evaluating the different factors responsible for female infertility and providing therapeutic management wherever feasible. The secondary objectivity of this study is to compare the findings of HSG with diagnostic laparoscopy and chromopertubation in evaluating the tubal factor of female infertility. On analyzing the type of infertility in our study, out of 130 couples, primary infertility was seen in $78.46 \%$ and secondary infertility was $22 \%$ which was very similar to the famous study of Duignan et al. ${ }^{7}$ The various causes causing female infertility were broadly categorized into ovarian, tubal, uterine, peritoneal, and cervical factors. Other causes like implantation failure, autoimmune disorders, psychological problems, and maturation defects of the 


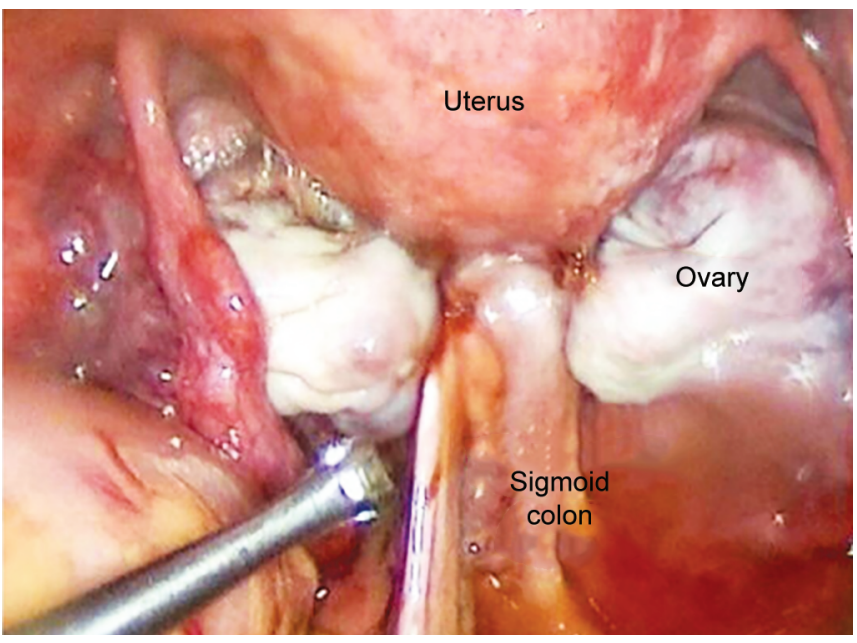

Fig. 6: Severe endometriosis grade IV with bilateral ovaries and sigmoid colon adhered to the posterior surface of the uterus- "Kissing Ovaries"

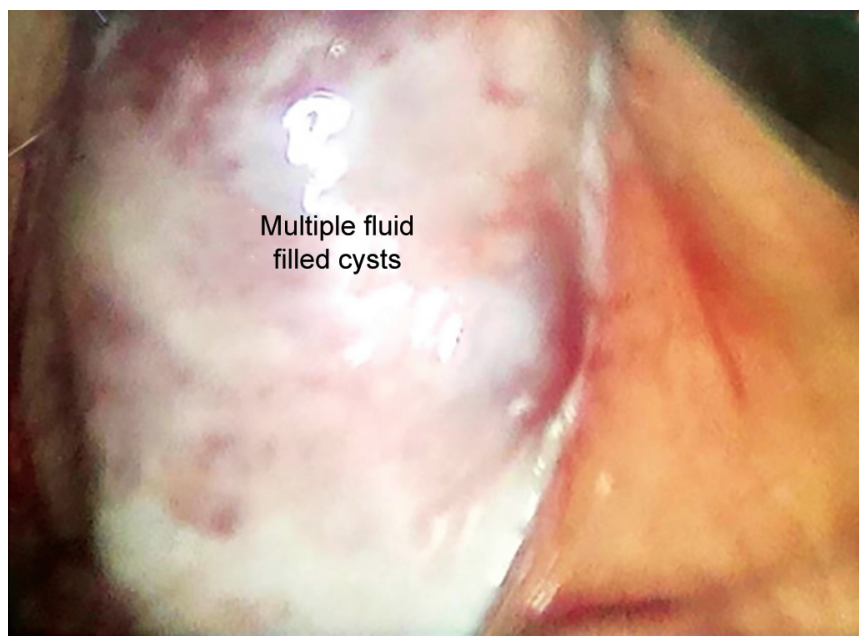

Fig. 7: Polycystic appearance of the ovary on laparoscopy

Table 2: Comparison of female factors of infertility by diagnostic laparoscopy in various studies

\begin{tabular}{|c|c|c|c|c|c|c|}
\hline Study and year & Tubal factor (\%) & Ovarian factor (\%) & Uterine factor (\%) & Peritoneal factor (\%) & Cervical factor (\%) & Multiple factors (\%) \\
\hline $\begin{array}{l}\text { Malekshah et al., } \\
2011^{8}\end{array}$ & 29.6 & 57.5 & 3.8 & 48.8 & - & - \\
\hline Sudha et al., $2013^{9}$ & 32.9 & 50.1 & 13.7 & - & - & - \\
\hline $\begin{array}{l}\text { Meuba et al., } \\
2014^{10}\end{array}$ & 32.5 & 35.6 & 31.9 & - & - & - \\
\hline Our study, 2017 & 68.4 & 51.5 & 24.6 & 33.8 & 1 & 93.84 \\
\hline
\end{tabular}

gametes were not evaluated and were well above the limits of our study.

In our study, we found that tubal factor causing infertility was present in $68.46 \%$, ovarian factor in $51.5 \%$, uterine factor $24.61 \%$, and peritoneal factor was present in $33.84 \%$ cases. Only 1 case $(0.77 \%)$ of cervical factor infertility was documented. The results of other studies are shown in Table 2 . There is significant variation in the percentage of various factors causing infertility in our study compared with the other studies, but one point has been common despite this variation that, there is a predominance of a female factor of infertility over all other factors individually. One hundred and twenty-two patients (93.84\%) out of 130 had more than one abnormality in laparoscopy. We noted in our study that the majority of patients were suffering from tubal factor infertility. Tubal appearance on laparoscopy was further subdivided into five different subcategories as beading of tubes, phimosis, hyperemia, hydrosalpinx, and lead pipe appearance, their impact on infertility was not known. Patency of the tube was assessed with chromopertubation during a diagnostic laparoscopy.

Not only our study but also every study ${ }^{8-10}$ we reviewed showed a marked preponderance of tubal blockage (unilateral or bilateral) as the most important factor for infertility. The majority of patients with ovarian factor infertility had polycystic ovarian syndrome (PCOS) 34 patients (26.15\%) (Fig. 7). Other causes of ovarian infertility in our study were ovarian cyst, low ovarian reserve with small or streak ovaries, and premature ovarian insufficiency. All the studies reviewed, demonstrated the dominance of PCOS in an ovarian factor of infertility. ${ }^{7-9}$ In our cases, 32 cases (24.61\%) contributed to uterine factors. The main cause of uterine factor infertility was uterine fibroid 11 cases (34.3\%). Three major causes of peritoneal factor infertility were endometriosis, pelvic adhesions, and pelvic tuberculosis in our study. There were a total of 44 cases (33.8\%) out of which 21 cases (47.7\%) had endometriosis and 19 cases had pelvic adhesions (43.18\%). There were 4 cases (9.09\%) of pelvic tuberculosis with dense peritoneal adhesions and tubo-ovarian mass, later confirmed with biopsy for granuloma. Most of the studies showed the dominance of endometriosis in the peritoneal factor of infertility, which was similar to our study. ${ }^{7-9}$ There was only 1 patient with cervical stenosis.

In our study, out of the 122 patients who were detected with abnormality through diagnostic laparoscopy, in 50 (38.46\%) patients therapeutic interventions were done in the same sitting. Out of the 11 patients diagnosed with fibroid uterus, 7 patients underwent myomectomy and 24 patients had PCOS drilling. Out of the 10 patients with an ovarian cyst, 7 patients underwent laparoscopic ovarian cystectomy. Other procedures like adhesiolysis, chocolate cyst drainage, and fimbrial dilatation were also done. We found in our study that, out of the 130 patients, only 90 patients $(69.23 \%)$ had similar findings of tubal patency on HSG and laparoscopic chromopertubation. The rest of the 40 patients had different results when their tubal patency was checked with chromopertubation. Therefore, the concordance between HSG and diagnostic laparoscopy was found out to be $69.23 \%$. This explains why HSG is only considered as a complementary investigation for tubal patency and not a substitution for laparoscopy.

In all patients, hysteroscopy was done along with diagnostic laparoscopy. Out of the 130 cases, 34 patients (26.15\%) had abnormalities. The most common finding on hysteroscopy was complete septum of the uterine cavity seen in 7 patients (20.5\%), followed by subseptate uterus in 6 patients (17.64\%). Other findings were submucous fibroid, endometrial polyp, and cavity adhesions. The therapeutic intervention was done in selected 


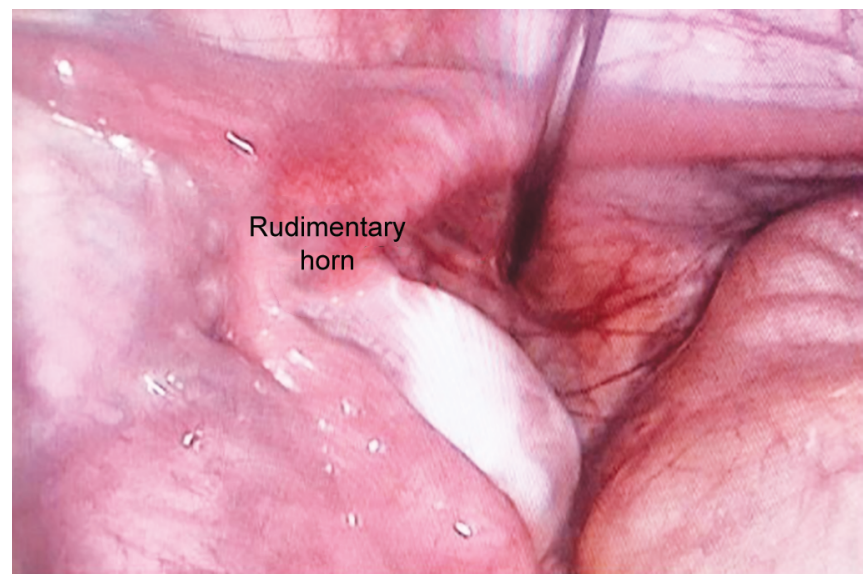

Fig. 8: Laparoscopic appearance of unicornuate uterus with a rudimentary horn

patients in the same or subsequent sitting. In 32 patients (24.16\%), the hysteroscopic therapeutic intervention was done. The most common procedure done was septal resection in 13 patients (40.62\%) and submucous fibroid removal in 7 patients (21.87\%). Other procedures like polyp removal, metroplasty, adhesiolysis, and tubal cannulation for the proximal tubal block were also done. Although a diagnosis of septate uterus per se is not an indication for septoplasty, many studies ${ }^{9,10}$ quoted their reproductive performance with an uncorrected septum is poor ( $80 \%$ pregnancy loss, $10 \%$ preterm delivery, $10 \%$ term delivery). Apart from the septate uterus, other uterine anomalies (Fig. 8) like bicornuate (2 cases) and unicornuate (4 cases) uterus were best diagnosed with hysterolaparoscopy.

Diagnostic hysterolaparoscopy is a very safe procedure. Other than mild abdominal pain, there were no major surgical or anesthetic complications in any of our patients.

In our study, the incidence of a male factor was only $10 \%$. This percentage seems to be less when compared to other studies (Table 2). This is because, for any couple with primary male factor infertility, found in semen analysis, intrauterine insemination (IUI) was the first line of management along with ovulation induction after tubal factors were ruled out by HSG. Severe male factor infertility patients were referred to an andrologist for evaluation and treatment. These female patients were deferred diagnostic laparoscopy, until three cycles of failed IUI. This is a selection bias in our study.

\section{CONCLUSION}

The results of our study suggest that diagnostic laparoscopy is the most useful procedure in the infertility workup. The different factors of infertility are thoroughly evaluated through laparoscopy, especially the peritoneal factors of which contributed to a large group of patients in our study, who were wrongly placed in the unexplained category, as they are correctable abnormalities that are unfortunately missed by routine pelvic examination and imaging procedures. The present study also indicates that the tubal patency plays a major role in conception and evaluation by laparoscopy along with chromopertubation, giving a better idea regarding the cause and management of the condition. This study reinstated the age-old theory that HSG and laparoscopy are complementary to each other and not a supplementary investigation in diagnosing tubal factor of infertility. Needless to emphasize that it is a very useful tool that can detect various structural abnormalities in the pelvis, tubes, uterus, and feasible therapeutic procedures can be done in the same sitting. When done by experienced hands with a proper patient selection of patients, hysterolaparoscopy can be considered as a definitive investigation and daycare procedure for evaluation of female infertility and formulating a specific plan of management.

\section{References}

1. Gnoth C, Godehardt E, Frank-Herrmann P, et al. Definition and prevalance of subfertility and infertility. Hum Reprod 2005;20(5):11441147. DOI: 10.1093/humrep/deh870.

2. Boivin J, Bunting L, Collins JA, et al. International estimates of infertility prevalance and treatment - seeking:potential need and demand for infertility medical care. Hum Reprod 2007;22(6):15061512. DOI: 10.1093/humrep/dem046.

3. Drake $T$, Tredway D, Buchanan G, et al. Unexplained infertility A reappraisal. Obstet Gynecol 1977;50:644-646.

4. Ganguly S, Unisa S. Trends of infertility and childlessness in India: findings from NFHS data. Facts Views Vis Obgyn 2010;2(2):131-138.

5. Tanahatoe SJ, Hompes PG, Lambalk CB. Investigation of the infertile couple. should diagnostic laparaoscopy be performed in the infertility work up program in patients undergoing intrauterine insemination? Hum Reprod 2003;18(1):8-11. DOI: 10.1093/humrep/ deg034.

6. Dupont J, Ngowa K, Nkongo K. Contribution of laparoscopy in the management of female fertility in low resource countries: a review of 208 cases at Yaoundev general hospital, Cameroon. Int J Clin Med 2015;6(12):934-999. DOI: 10.4236/ijcm.2015.612122.

7. Duignan NM, Jordan JA, Coughlan BM, et al. One thousand consecutive cases of diagnostic laparoscopy. BJOJ: An International Journal Obstetrics Gynaecology 1972;79(11):1016-1024. DOI: 10.1111/ j.1471-0528.1972.tb11880.x.

8. Malekshah AK, Moghaddam AE, Moslemizadeh N, et al. Infertility in Mazandaran province - north of Iran: an etiological study. Iranian J Reprod Med 2011;9(1):21-24.

9. Sudha G, Reddy K. Causes of female infertility: a cross-sectional study. Int J Lat Res Sci Technol 2013;2(6):119-123.

10. Meuba IE, Ugwu EO, Obi SN, et al. Clinical management and therapeutic outcome of infertile couples in south east Nigeria. Ther Clin Risk Manag 2014;10:763. 